\title{
How warm is the molecular gas in active environments?
}

\author{
Stefanie Mühle* \\ Joint Institute for VLBI in Europe \\ Postbus 2, 7990 AA Dwingeloo, The Netherlands \\ E-mail: muehledjive.nl

\section{Christian Henkel} \\ Max-Plank-Institut für Radioastronomie \\ Auf dem Hügel 69, 53121 Bonn, Germany \\ E-mail: p220henempifr-bonn.mpg.de

\section{Tahlia de Maio} \\ University of Colorado \\ 389 UCB, Boulder CO, 80309, USA \\ E-mail: tahlia.demaio@colorado.edu
}

\section{Ernest R. Seaquist}

Department of Astronomy and Astrophysics, University of Toronto

50 St. George Street, Toronto, ON M5S 3H4, Canada

E-mail: seaquist@astro.utoronto.ca

The question whether or not the initial mass function is universal, i.e. the same in all kinds of environments, is of critical importance for the theory of star formation and still intensely debated. A top-heavy initial mass function may be the result of star formation out of dense molecular clouds with a temperature of $\sim 100 \mathrm{~K}$. Such a molecular gas phase is not commonly found in the Galactic plane, but may be present in active environments like cores of starburst galaxies or AGN. Unfortunately, the kinetic temperature of the molecular gas in external galaxies is often not well constrained. Having proven the diagnostic power of selected formaldehyde lines as tracers of the properties of the molecular gas in external galaxies, we have engaged in observing these diagnostic lines in a number of starburst galaxies and near AGN. This contribution presents the latest results of these studies.

10th European VLBI Network Symposium and EVN Users Meeting: VLBI and the new generation of radio arrays

September 20-24, 2010

Manchester, UK

\footnotetext{
${ }^{*}$ Speaker.
} 


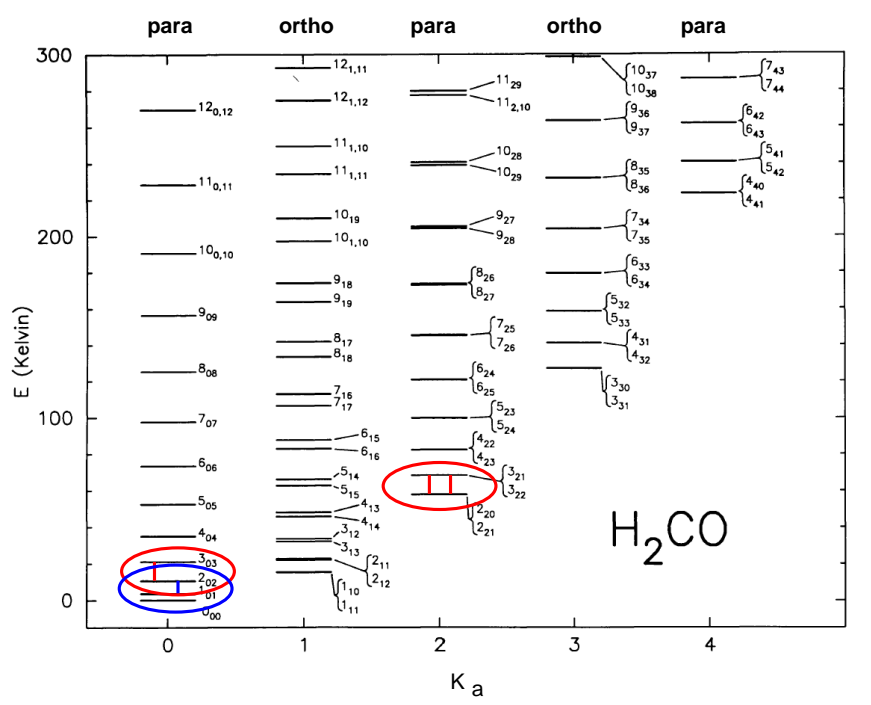

Figure 1: The lowest energy levels of ortho- and paraformaldehyde, adapted from [7]. The para- $\mathrm{H}_{2} \mathrm{CO}$ transitions at $218 \mathrm{GHz}$ are marked in red, the diagnostic para- $\mathrm{H}_{2} \mathrm{CO}$ transition at $146 \mathrm{GHz}$ in blue.

\section{Is the initial mass function universal?}

One of the fundamental debates in the field of star formation is the question whether the stellar initial mass function (IMF) is universal or not. A number of recent observations have been interpreted as evidence for a top-heavy initial mass function, spanning a variety of objects, from the centre of our Galaxy to circumnuclear starburst regions and ultra-compact dwarf galaxies (e.g. [3,4], see also [1] for a critical review). Hydrodynamical simulations can reproduce such a topheavy IMF if the raw material of star formation, the dense molecular gas, is assumed to have a kinetic temperature of $\sim 100 \mathrm{~K}[6]$. Such a temperature is significantly higher than what is observed in the dense starless cloud cores of the Galactic plane, but is similar to kinetic temperatures that have been derived for a number of starburst galaxies and AGN from observations of highly excited ammonia lines and IR rotational $\mathrm{H}_{2}$ lines $[10,14]$. In a starburst galaxy, the conversion factor between the $\mathrm{CO}(1-0)$ line intensity and the $\mathrm{H}_{2}$ column density can be up to an order of magnitude smaller than the standard conversion factor that has been derived empirically in the Galactic plane. Thus, the properties of the molecular gas in active environments like the cores of starburst galaxies or AGN, whose activity leads to strong feedback in the form of intense UV radiation, X-ray and cosmic ray emission, seem to differ significantly from the properties of the molecular gas in the Galactic disk. This may be true even in the densest cores [13].

\section{Formaldehyde lines as extragalactic diagnostics}

Unfortunately, the kinetic temperature of the molecular gas in most external galaxies is not well constrained yet, because many of the most common tracer molecules suffer from a degeneracy between the kinetic temperature and the gas density. Instead, the kinetic temperature of the molecular gas is often set to be similar to the dust temperature, an assumption that may be valid 

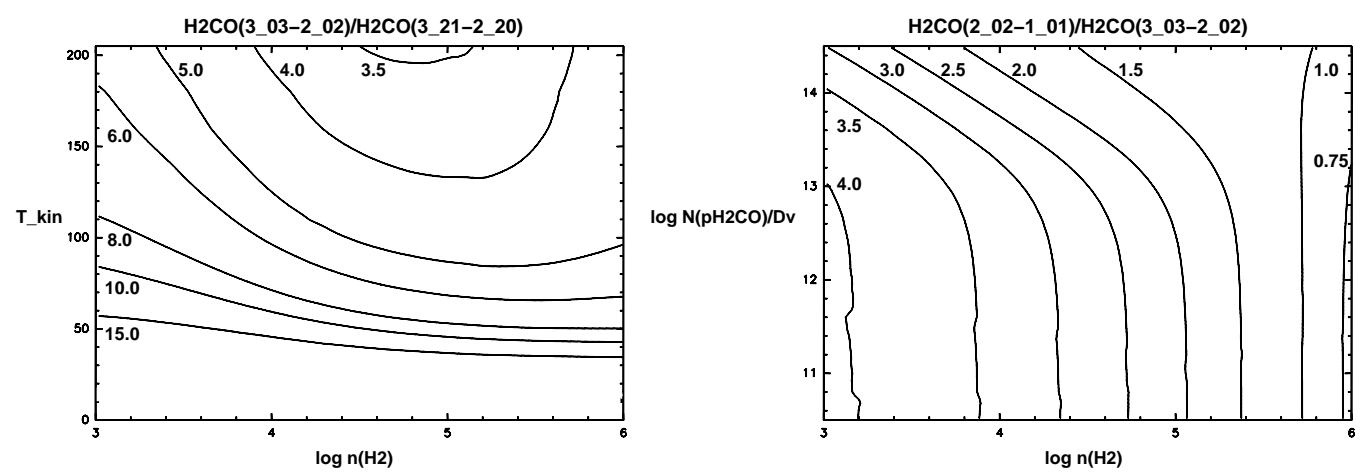

Figure 2: Plots derived from our LVG code illustrating the diagnostic value of the selected line ratios: a) The interladder line ratio $\mathrm{H}_{2} \mathrm{CO}\left(3_{03} \rightarrow 2_{02}\right) / \mathrm{H}_{2} \mathrm{CO}\left(3_{21} \rightarrow 2_{20}\right)$ traces the kinetic temperature with only a weak dependence on the gas density or the para- $\mathrm{H}_{2} \mathrm{CO}$ column density (here: $N_{\mathrm{pH} 2 \mathrm{CO}} / \Delta v=10^{12} \mathrm{~cm}^{-2} / \mathrm{km} \mathrm{s}^{-1}$ ). b) At a given kinetic temperature (here: $T_{\text {kin }}=100 \mathrm{~K}$ ), the intraladder line ratio $\mathrm{H}_{2} \mathrm{CO}\left(2_{02} \rightarrow 1_{01}\right) / \mathrm{H}_{2} \mathrm{CO}\left(3_{03} \rightarrow\right.$ $2_{02}$ ) provides an excellent estimate of the gas density with only a moderate dependence on the para- $\mathrm{H}_{2} \mathrm{CO}$ column density.

in the densest, most FUV-shielded cores of giant molecular clouds, but can only be a lower limit in most other cases. Ammonia, a molecule that thanks to its symmetric top structure is well suited as a temperature probe, serves well as the standard "cloud thermometer" in our Galaxy where the difference between the derived rotational temperature and the kinetic temperature is small. Unfortunately, the fractional abundance of ammonia varies between $10^{-5}$ in hot cores [9] and $10^{-8}$ in dark clouds [2], which makes this molecule less suitable as a tracer in extragalactic observations that by default average over a significant part of a galaxy.

In contrast, formaldehyde $\left(\mathrm{H}_{2} \mathrm{CO}\right)$, an only slightly asymmetric top, shows little variation in its fractional abundance in a variety of galactic environments, with infrared sources showing the largest discrepancy with a factor of $5-10$ [5]. The two subspecies para- $\left(K_{a}=0,2,4, \ldots\right)$ and orthoformaldehyde $\left(K_{a}=1,3,5, \ldots\right)$ possess a rich spectrum with a number of transition lines in the cm- and mm-range that can be observed with existing telescopes (Fig. 1). On the other hand, these radiative transitions are not so numerous that line blending, which is commonly found in complex molecules, is a major concern. As a rule of thumb, line intensity ratios that involve different $K_{a}$ ladders (interladder ratios) are good tracers of the kinetic temperature, while intraladder ratios, i.e. ratios of transitions of the same $K_{a}$ ladder, sensitively probe the gas density once the temperature is determined (Fig. 2, [7]).

\section{The project}

Considering the important role of the kinetic temperature of the star-forming molecular gas in active environments on one hand and the scarcity of reliable temperature measurements in external galaxies on the other hand, we have engaged in deriving the properties of the dominant phase of the molecular gas in a number of nearby starburst galaxies and AGN using the diagnostic properties of paraformaldehyde lines at $146 \mathrm{GHz}$ and at $218 \mathrm{GHz}$. The paraformaldehyde lines at $218 \mathrm{GHz}$ are particularly valuable from a diagnostic point of view. Within $1 \mathrm{GHz}$, i.e. usually within the same low frequency resolution spectrum, there are the three para- $\mathrm{H}_{2} \mathrm{CO}$ tran- 

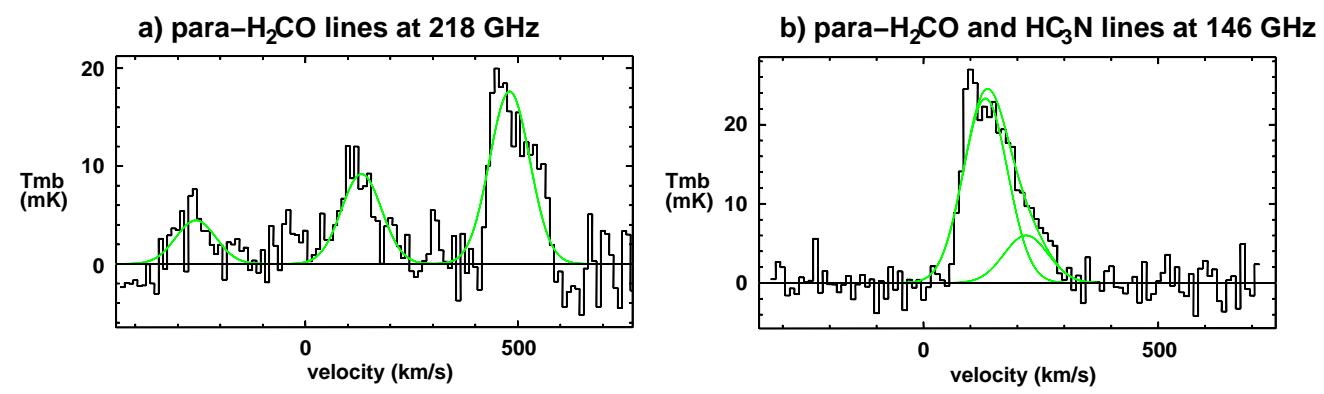

Figure 3: Paraformaldehyde lines observed towards the SW lobe of $\mathrm{M} 82$. a) Para- $\mathrm{H}_{2} \mathrm{CO}$ lines at $218 \mathrm{GHz}$. The velocity scale of the spectrum refers to the $\mathrm{H}_{2} \mathrm{CO}\left(3_{22} \rightarrow 2_{21}\right)$ line at $132 \mathrm{~km} \mathrm{~s}^{-1}$. The $\mathrm{H}_{2} \mathrm{CO}\left(3_{03} \rightarrow 2_{02}\right)$ and the $\mathrm{H}_{2} \mathrm{CO}\left(3_{21} \rightarrow 2_{20}\right)$ transitions are offset by $348.5 \mathrm{~km} \mathrm{~s}^{-1}$ and $-389.7 \mathrm{~km} \mathrm{~s}^{-1}$, respectively. b) In the $146 \mathrm{GHz}$ spectrum, the $\mathrm{H}_{2} \mathrm{CO}\left(2_{02} \rightarrow 1_{01}\right)$ line at $132 \mathrm{~km} \mathrm{~s}^{-1}$ is blended with the $\mathrm{HC}_{3} \mathrm{~N}(16 \rightarrow 15)$ line, which is offset by $86.46 \mathrm{~km} \mathrm{~s}^{-1}$ from the $\mathrm{H}_{2} \mathrm{CO}\left(2_{02} \rightarrow 1_{01}\right)$ transition. The green curves show the Gaussian fit to each individual line as well as the superposition of all fitted Gaussians.

sitions $3_{03} \rightarrow 2_{02}(218.22 \mathrm{GHz}), 3_{22} \rightarrow 2_{21}(218.48 \mathrm{GHz})$ and $3_{21} \rightarrow 2_{20}(218.76 \mathrm{GHz})$ (Fig. 3a). While the $\mathrm{H}_{2} \mathrm{CO}\left(3_{22} \rightarrow 2_{21}\right)$ line may be contaminated by methanol emission, the intensity ratio of the other two lines forms an excellent temperature tracer, without any uncertainty due to calibration issues, pointing errors or different beam widths. The set of diagnostic lines is completed by $\mathrm{H}_{2} \mathrm{CO}\left(2_{02} \rightarrow 1_{01}\right)$ at $145.60 \mathrm{GHz}$, an easily detectable line that together with $\mathrm{H}_{2} \mathrm{CO}\left(3_{03} \rightarrow 2_{02}\right)$ forms a sensitive density tracer.

The reasonable assumption that the emission of all the $\mathrm{H}_{2} \mathrm{CO}$ lines originates from the same volume implies that the lines have the same velocity profile, thus considerably reducing the number of free parameters in the derivation of the line intensities: If the line profiles are approximated by Gaussian curves, the velocity and width of all lines should match, leaving the peak intensity as the only free parameter. Thus, by fixing the velocity and the width of the common line profile with the help of a strong line, even the intensities of weak (Fig. 3a) or blended lines (Fig. 3b) can be derived with high reliability.

By comparing the line intensity ratios with the predicted ratios of a non-LTE model physical properties of the gas like the kinetic temperature and the average gas density within the molecular cloud complexes can be derived. For the analysis of the para- $\mathrm{H}_{2} \mathrm{CO}$ lines, we have developed a model with a spherically symmetric cloud geometry and adopted the LVG approximation. The searched parameter space covers a kinetic temperature of $T_{\text {kin }}=5$ to $300 \mathrm{~K}$ in steps of $5 \mathrm{~K}$, a molecular gas density of $\log n_{\mathrm{H} 2}=3.0$ to $6.0\left(\right.$ in $\left.\mathrm{cm}^{-3}\right)$ in steps of 0.1 and a para- $\mathrm{H}_{2} \mathrm{CO}$ column density per velocity interval of $\log N_{\mathrm{pH} 2 \mathrm{CO}} / \Delta v=10.5$ to $14.5\left(\mathrm{in} \mathrm{cm}^{-2} \mathrm{~km}^{-1} \mathrm{~s}\right.$ ) in steps of 0.1 (see [11] for details).

\section{First results}

The first results of our studies support the view that a considerable fraction of the dense molecular gas in starburst galaxies is significantly warmer than the dense molecular gas in quiescent 

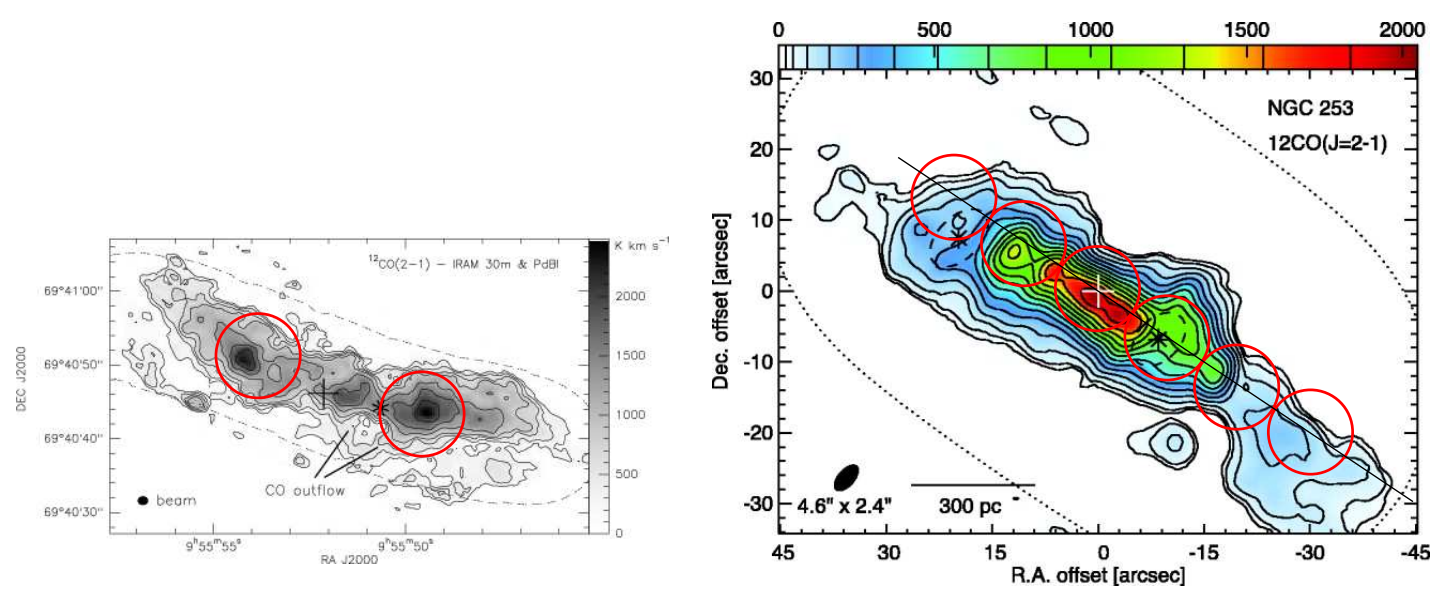

Figure 4: Positions of observed $\mathrm{H}_{2} \mathrm{CO}$ emission in M 82 (left) and NGC 253 (right) superposed on highresolution maps of the integrated $\mathrm{CO}(2 \rightarrow 1)$ intensity adapted from [17] and [15], respectively. The diameter of the red circles corresponds to the width of the beam of the IRAM $30-\mathrm{m}$ telescope at $218 \mathrm{GHz}$.

galaxies. A comparison between M 82 and NGC 253 shows interesting similarities and differences. Both galaxies are considered to be prototypical starburst galaxies, nearby and seen at high inclination, with high infrared luminosities, super-star clusters and a prominent galactic wind. In both galaxies, the dense molecular gas is concentrated around the starbursting nucleus. The fractional abundances of a number of molecules, though, differ strongly between the two galaxies, which is seen as evidence that the starburst in M 82 is in an evolved state while the starburst in NGC 253 is (kept) young. Note that the massive star cluster M82F is to date the best candidate to host a top-heavy IMF [1].

We observed the two lobes of the circumnuclear ring in M 82 and six positions along the major axis of the circumnuclear disk of NGC 253 with the IRAM 30-m telescope both at $218 \mathrm{GHz}$ and at $146 \mathrm{GHz}$ to an rms noise level of a few milliKelvin at a velocity resolution of $10 \mathrm{~km} \mathrm{~s}^{-1}$ at each frequency and pointing position (Fig. 4). The $\mathrm{H}_{2} \mathrm{CO}\left(2_{02} \rightarrow 1_{01}\right)$ line at $145.60 \mathrm{GHz}$, slightly blended with the $\mathrm{HC}_{3} \mathrm{~N}(16 \rightarrow 15)$ line, has been detected at all pointing positions, while the three $\mathrm{H}_{2} \mathrm{CO}$ lines at $218 \mathrm{GHz}$ have been deteced at all positions except for the two outermost pointings in NGC 253. As an example, the spectra of the southwestern lobe in M 82 are shown in Fig. 3.

The intensity ratios derived from the observed lines are remarkably similar in the two lobes of M 82, whereas the ratios vary significantly along the major axis of the molecular disk in NGC 253, indicating different physical properties of the dense molecular gas across the galactic core region. In M 82, the LVG analysis suggests a kinetic temperature of $T_{\text {kin }} \sim 200 \mathrm{~K}$, a gas density of $n_{\mathrm{H} 2} \sim$ $7 \cdot 10^{3} \mathrm{~cm}^{-3}$ and a molecular gas mass of $\sim 3 \cdot 10^{8} M_{\odot}$ in the lobes, which is in good agreement with the high-excitation molecular gas component of other recent molecular line studies, which may constitute more than half of the total molecular gas mass in M 82 [8,16]. A preliminary analysis of the line ratios derived for the different positions in NGC 253 yields kinetic temperatures of $T_{\text {kin }} \sim 70 \ldots 150 \mathrm{~K}$ and gas densities of $n_{\mathrm{H} 2} \sim 5 \ldots 25 \cdot 10^{3} \mathrm{~cm}^{-3}$. Thus, in both galaxies, the dense molecular gas component traced by paraformaldehyde lines is considerably warmer than the dust and in fact warm enough to give rise to a top-heavy IMF should this gas be turned into stars. 


\section{Outlook}

The survey of the temperature of dense molecular gas in active extragalactic environments is still in an early phase and many questions about the extent of the warm gas phase and the environments where it can be found remain. ALMA will provide a boost in sensitivity which will make the para- $\mathrm{H}_{2} \mathrm{CO}$ lines readily detectable in a variety of external galaxies, while at the same time offering unprecedented angular resolution. This will provide information on the distribution of the traced molecular gas phase and constrain source sizes. In the cm-range, e-MERLIN may be used to supply high-resolution maps of the ortho- $\mathrm{H}_{2} \mathrm{CO} K$-doublet transition $1_{10} \rightarrow 1_{11}$, which will allow us to derive the ortho-to-para formaldehyde ratio, thus providing information on the temperature of the dust grains at the time when the formaldehyde molecules were formed.

\section{Acknowledgments}

We wish to thank the staff at Pico Veleta/Granada for their support during the observations. E.R.S. acknowledges a Discovery Grant from NSERC. This work has made use of the following software and resources: GILDAS, specx, Statistiklabor (FU Berlin, CeDiS), asyerr (Seaquist \& Yao), the JPL Catalog of spectral lines, The Cologne Database for Molecular Spectroscopy [12] and NASA's Astrophysics Data System Bibliographic Services (ADS).

\section{References}

[1] Bastian, N., Covey, K.R., \& Meyer, M.R. 2010, ARAA 48, 339

[2] Benson, P. J., \& Myers, P. C. 1983, ApJ, 270, 589

[3] Dabringhausen, J., Kroupa, P, \& Baumgardt, H. 2009, MNRAS 394, 1529

[4] Habergham, S.M., Anderson, J.P., \& James, P.A. 2010, ApJ 717, 342

[5] Johnstone, D., Boonman, A.M.S., \& van Dishoeck, E.F. 2003, A\&A 412, 157

[6] Klessen, R.S., Spaans, M., \& Jappsen, A.K. 2007, MNRAS 374, L29

[7] Mangum, J.G. \& Wootten, A. 1993, APJS 89, 123

[8] Mao, R.Q., Henkel, C., Schulz, A., et al. 2000, A\&A 358, 433

[9] Mauersberger, R., Henkel, C., \& Wilson, T.L. 1987, A\&A 173, 352

[10] Mauersberger, R., Henkel, C., Weiß, A., et al. 2003 A\&A 403, 561

[11] Mühle, S., Seaquist, E.R., \& Henkel, C. 2007, ApJ 671, 1579

[12] Müller, H.S.P., Schlöder, F., Stutzki, J., et al. 2005 J. Mol. Struct. 742, 215

[13] Papadopoulos, P.P. 2010, ApJ 720, 226

[14] Rigopoulou, D., Kunze, D., Lutz, D., et al. 2002, A\&A 389, 374

[15] Sakamoto, K., Ho, P.T.P., Iono, D., et al. 2006, ApJ 636, 685

[16] Ward, J.S., Zmuidzinas, J., Harris, A.I., et al. 2003, ApJ 587, 171

[17] Weiß, A., Neininger, N., Hüttemeister, S., et al. 2001, A\&A 365, 571 